\title{
Tip-timing analysis of last stage steam turbine mistuned bladed disc during run-down
}

\author{
Romuald Rzadkowski ${ }^{1,2 *}$, Leszek Kubitz ${ }^{1}$, Michat Maziarz ${ }^{1}$, Pawel Troka ${ }^{1}$, Leszek \\ Piechowski ${ }^{1}$ and Ryszard Szczepanik ${ }^{2}$ \\ ${ }^{1}$ Institute of Fluid-Flow Machinery Polish Academy of Sciences, Fiszera 14, 80-952 Gdansk, Poland \\ ${ }^{2}$ Air Force Institute of Technology, Księcia Bolesława, 6, 01-494 Warsaw, Poland
}

\begin{abstract}
This paper presents the experimental and numerical studies of last stage LP mistuned steam turbine bladed discs during run-down. The natural frequencies and mode shapes of the turbine bladed disc were calculated using an FE model. The influence of shaft was considered. The tip-timing method was used to find the mistuned bladed disc modes and frequencies. The numerical results were compared with experimental ones.
\end{abstract}

\section{Introduction}

In the most commonly used low pressure steam turbines, during certain summer exploitation conditions, a problem concerning high level vibrations in unshrouded last stage low pressure blades was reported. In Rzadkowski et al. [1] different models of bladed discs were assumed. The first one was simply a bladed disc without a shaft and the second was a bladed disc with part of the shaft. In this paper a shaft with bladed discs, will be considered to analyse multistage coupling and the influence of the shaft on mistuned modes. Rzadkowski and Drewczynski [2] analysed the forced vibration results for steam turbine rotors with 8 tuned bladed discs, each with different numbers of blades. The multistage coupling appeared in modes with up to one nodal diameter. Rzadkowski and Maurin [3] analysed the free and forced vibration results for steam turbine rotors with 8 mistuned bladed discs, each with different numbers of blades. The mistuning distorted the nodal diameters of modes mainly in the unshrouded bladed discs and caused multistage coupling between various nodal diameters. Rzadkowski et al. [1] found that the LP steam turbine last stage bladed disc is very sensitive to any kind of mistuning. Even the smallest changes in the natural frequencies of blades distort nodal diameters. A numerical study of a steam turbine bladed disc with real, small mistuning showed that nodal diameters do not appear in the first mode family associated with the natural frequencies of a cantilever blade and only individual blades vibrate. Numerical analyses require experimental verification. Przysowa et al. [4] presented a tip-timing system and its measurements of last stage rotor blades, taken in a vacuum spin chamber. These measurements were of only the rotor with bladed discs during run-down A review of tip-timing models is presented in Rzadkowski et al. [6]. In this paper, we will analyse numerically and experimentally the dynamics of mistuned bladed discs during rundown in a vacuum spin chamber. The numerical model will be of the full shaft. The numerical

\footnotetext{
*Corresponding author: z3@imp.gda.pl
} 
results will be compared with the tip-timing measurements of mistuned bladed disc frequencies and modes.

\section{Experimental results}

An LP last stage mistuned bladed disc on a rotor was tested in a vacuum spin chamber to measure blade vibration (Fig. 1). The blade displacements were measured during run-down (Figs. 2-4). Fig. 2 presents the 53 blade tip displacements during a run-down from $3052 \mathrm{rpm}$ to $492 \mathrm{rpm}$. In order to prevent excessive blade stress through self-excitation, the geometries of the blades were deliberately mistuned by removing a thin layer of material from around the tip of every second blade (feathering). Blade feathering caused a number of frequency groups, presented in Fig. 2, for various engine order (EO) excitations connected with blade natural frequencies (Fig. 6). Fig. 3 (an enlarged section of Fig. 2) presents 27 blade tip displacements during a run-down from 1958 to $1878 \mathrm{rpm}$ for 3EO. Here, we see that tiptiming can measure blade damping in the flow.

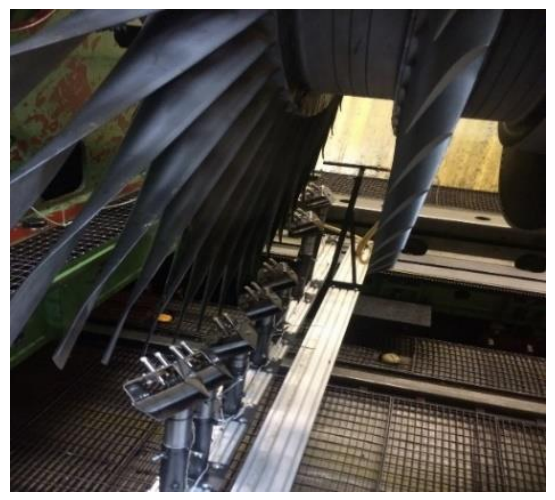

Fig. 1. View of the rotor in the vacuum spin chamber

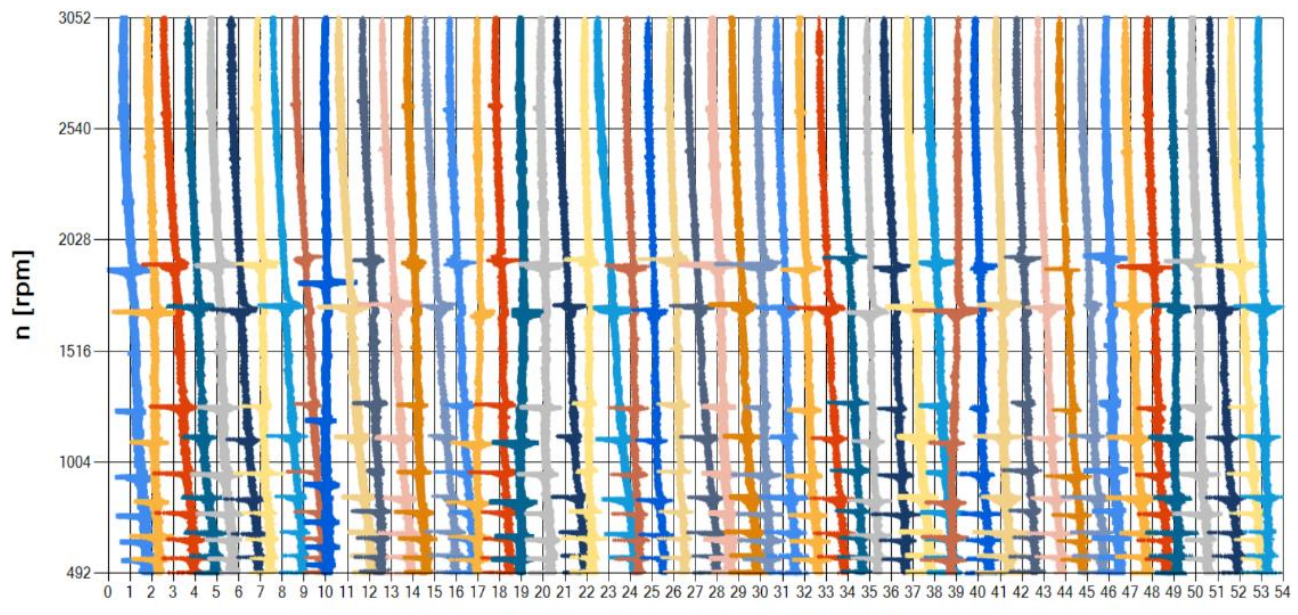

[Blade number + amplitude $3 \mathrm{~mm} / 1$ ]

Fig. 2. Tip displacements of 53 rotor blades during run-down, 3052 - 492 rpm 


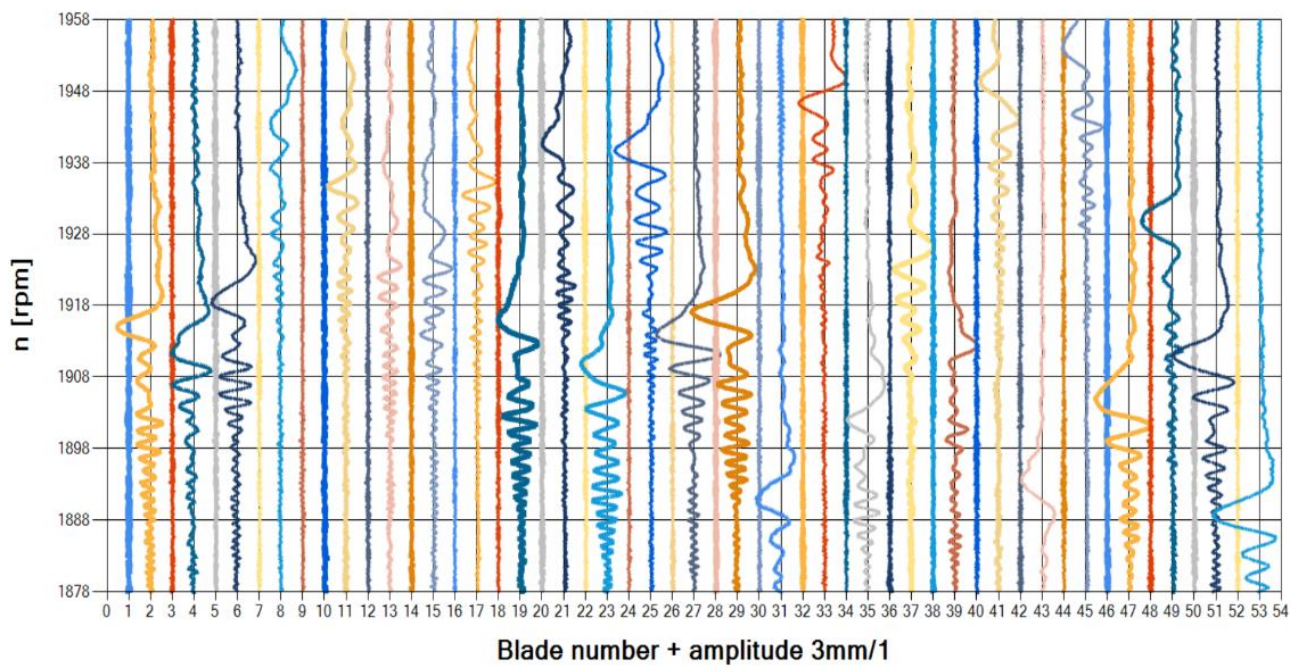

Fig. 3. Tip displacements of 53 rotor blades during run-down, $1958-1878 \mathrm{rpm}$

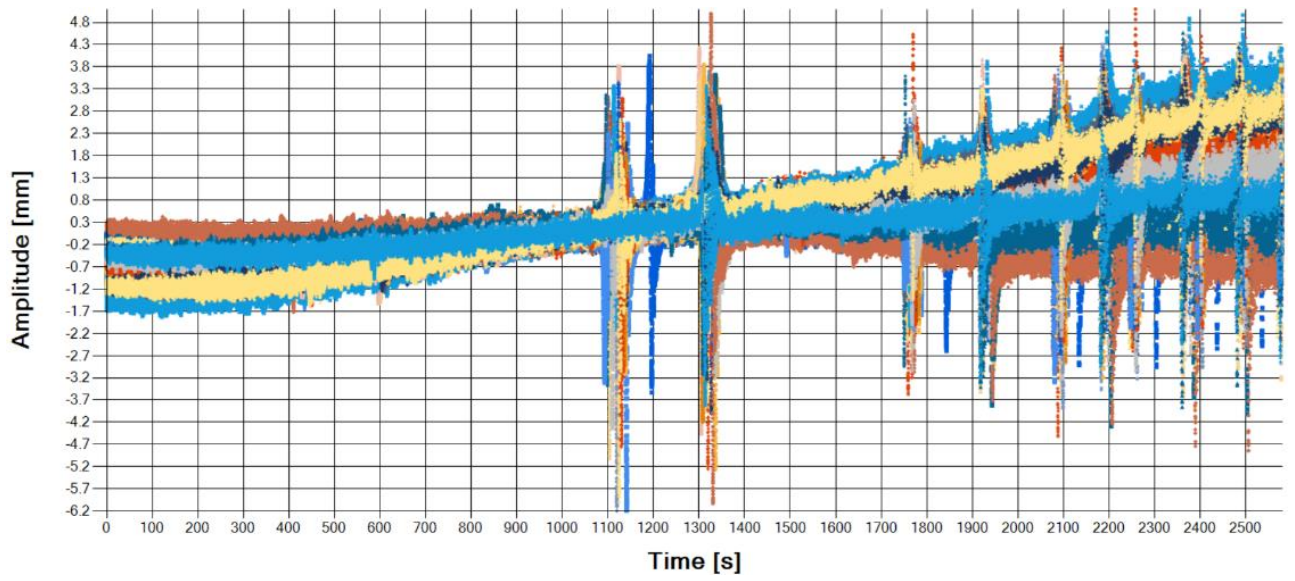

Fig. 4. Tip displacements of 53 rotor blades during run-down, 3052 - $492 \mathrm{rpm}$

Fig. 4 presents the displacements of all the 53 blades during run-down measured. This shows that during the run-down, the maximal amplitude is $4.46 \mathrm{~mm}$. Figs. 2-4 present the measurements from only one sensor, which were affected by aliasing. In order to obtain the blade amplitudes, phases and frequencies the linear sine fitting was used on the basis of signals from several sensors. The blade frequencies and amplitudes for $3 \mathrm{EO}$ excitation obtained from tip-timing analysis (3 sensors) are presented in Fig. 5. The 3EO resonance is seen in the Campbell diagram of the rotor blade (Fig. 6) and Fig. 3. In 3EO the rotor blades vibrated with maximal amplitudes (Fig. 6). 


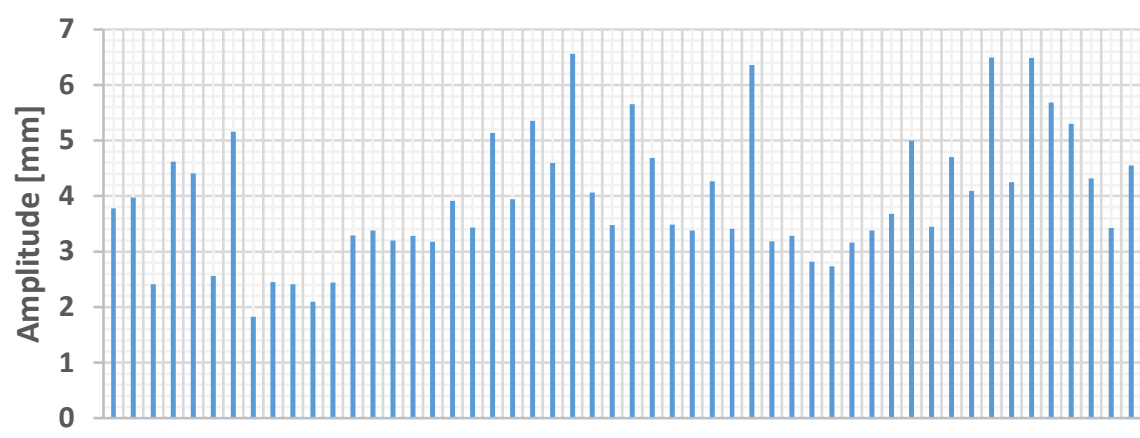

13355799111315171921232527293133353740424446485052 Blade number [-]

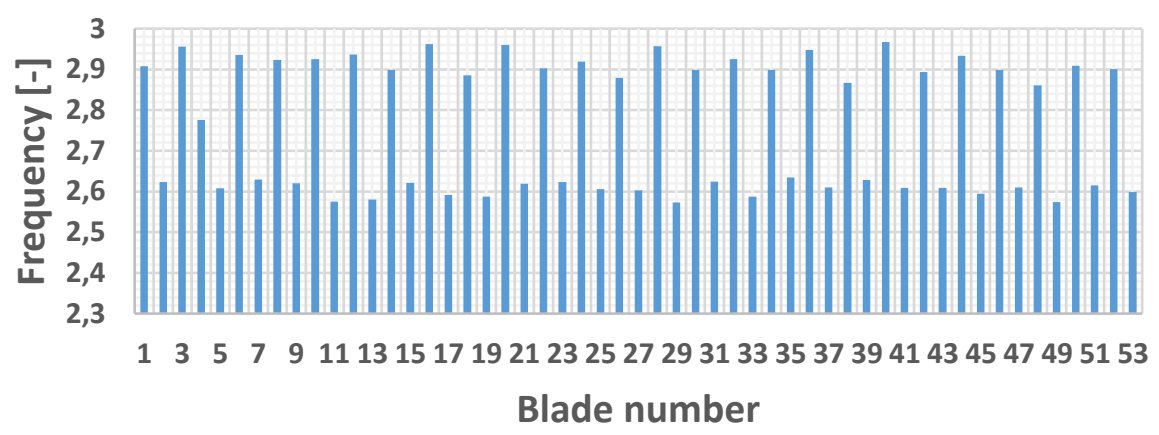

Fig. 5. 53 rotor blade amplitudes and frequencies for 3EO resonance

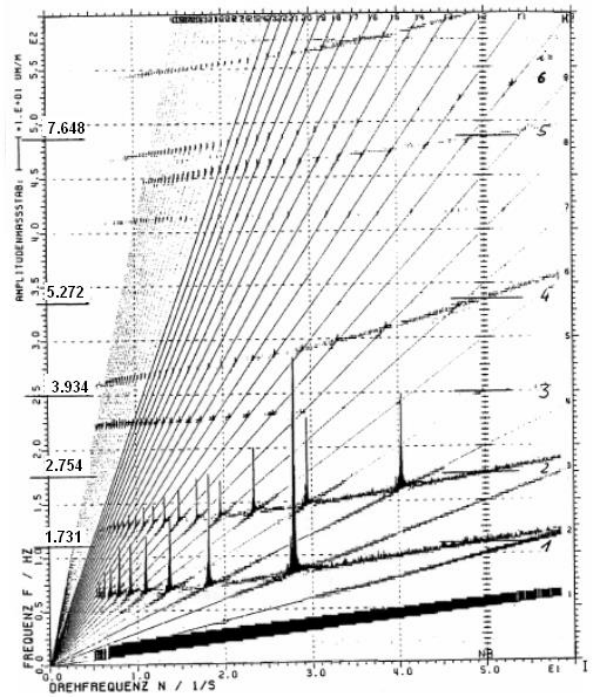

Fig. 6. Campbell diagram of rotor blade 


\section{Numerical results}

A shaft with bladed discs was considered to analyse the frequencies of multistage coupling and the influence of the shaft on mistuned modes. Blade mistuning was introduced to the numerical model by changing the FE model of particular rotor blades (Fig. 5). Three groups of frequencies were assumed: without feathering (2.93-2.89 - non-dimensional frequency, 26 blades), with feathering (2.62-2.57, 26 blades) and blade 4 (2.77). There was less than 10 $\mathrm{Hz}$ mistuning difference between groups with and without fathering. The tip-timing measurements show that in the first group $(2.93-2.89,26)$ without feathering and also in the group with feathering (2.62-2.57, 26 blades) another mistuning appeared as the result of a manufacturing error (Fig. 5).

\section{Comparison of numerical and experimental results}

The tip-timing measurements give the frequencies and amplitudes of the mistuned bladed disc modes. Fig. 7 compares several experimentally measured (left) and numerically (right) calculated mistuned bladed disc modes. The comparison is satisfactory. The discrepancies result from the fact that the numerical calculations assumed a rigid support of the full shaft in bearings instead of the stiffness and bearing damping coefficient. The results show that nodal diameters do not appear in the first mode family associated with the natural frequencies of a cantilever blade and only individual blades vibrate.
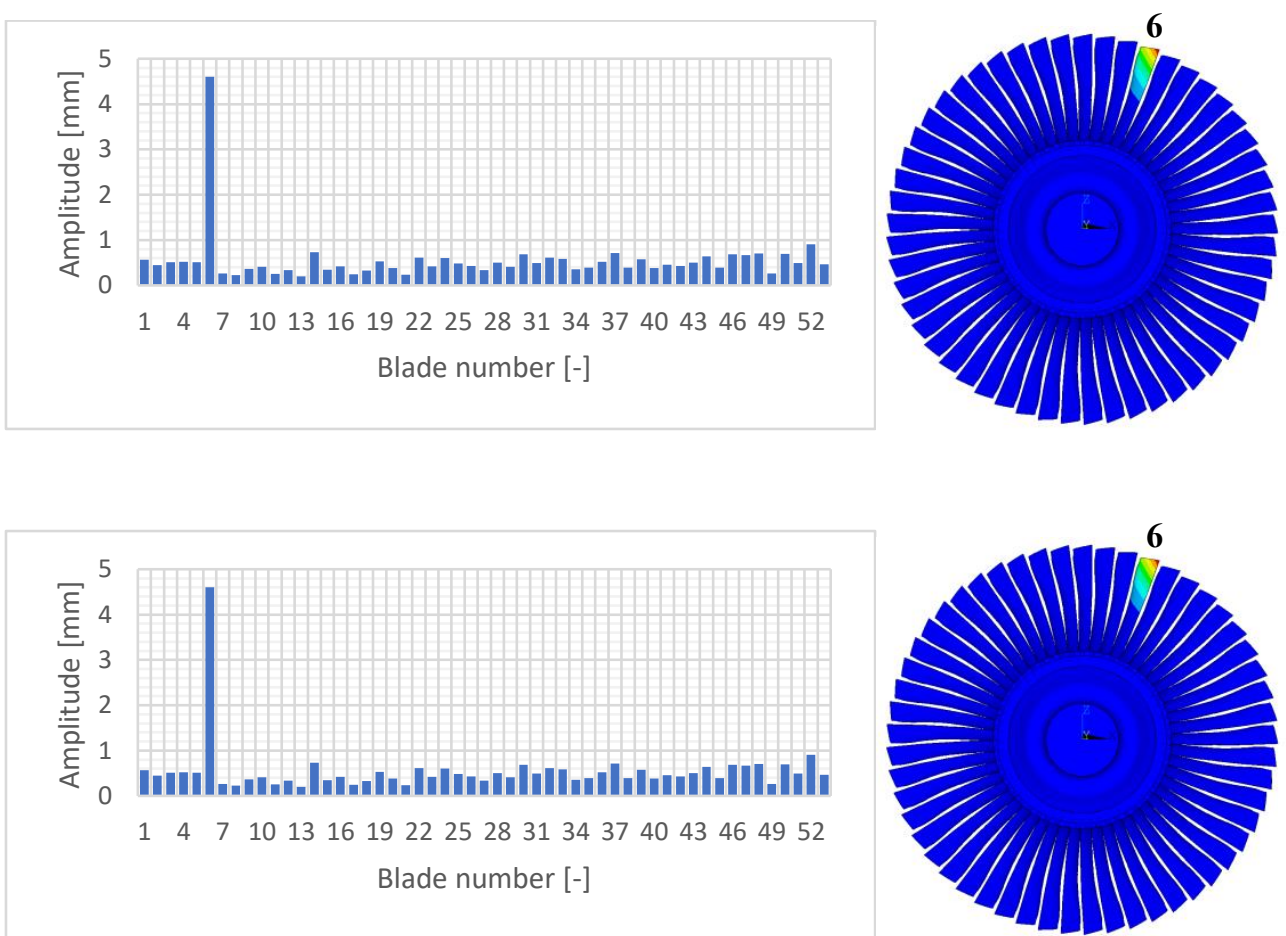

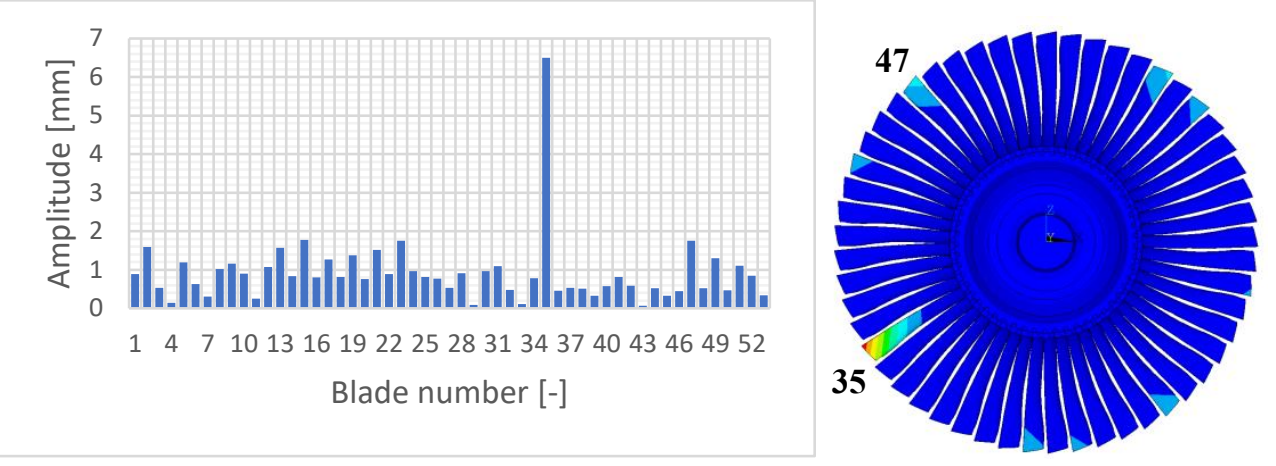

Fig. 7. Experimentally and numerically obtained mistuned bladed disc modes

\section{Conclusions}

This paper has shown the vibration modes of a LP mistuned last stage bladed disc. Complete shaft with four bladed discs was created to analyse multistage coupling and the influence of the whole shaft on mistuned bladed disc modes.

Multistage coupling through the rotor influences only bladed discs with zero- and onenodal-diameter modes. This increases the number of resonances and changes the Campbell diagram. The blade mistuning pattern was measured by tip-timing in a vacuum spin-chamber. The experimental results from the tip-timing analysis show that that nodal diameters do not appear in the first mode family associated with the natural frequencies of a cantilever blade and only individual blades vibrate. The numerical results confirm this.

This research has been financed by Polish Ministry of Science (NCBiR) funds for the years 20122017 as development project PBS1/B4/5/2012. All the numerical calculations were carried out at the Academic Computer Centre TASK (Gdansk, Poland).

\section{References}

[1] R. Rzadkowski, A. Maurin, L. Kubitz, R. Szczepanik, (2016), Forced Vibration of Mistuned Bladed Disc in Last Stage LP Steam Turbine, Proceedings of ASME Turbo Expo 2016, GT2016-57427 (2016).

[2] R. Przysowa, R. Spychała, P. Majewski, E. Rokicki E., Experimental Analysis of Rotor Blades in Vacuum Chamber, in book Ed Rzadkowski R., Szczepanik R., Dynamics of the Last Stage Low Pressure Steam Turbine Rotor Blades, 2017 ITWL Warsaw (2017).

[3] R. Przysowa, R. Spychała, P. Majewski, E. Rokicki, Monitoring of Blade Vibration in a Steam Turbine Power Station, in book Ed. Rzadkowski R., Szczepanik R., Dynamics of the Last Stage Low Pressure Steam Turbine Rotor Blades, 2017 ITWL Warsaw (2017).

[4] R. Rzadkowski, E. Rokicki, L. Piechowski, R. Szczepanik, Mechanical Systems and Signal Processing, 76-77, 213-227, (2016). 